Check for updates

Cite this: RSC Adv., 2017, 7, 51452

Received 5th September 2017 Accepted 30th October 2017

DOI: $10.1039 / c 7 r a 09865 k$

rsc.li/rsc-advances

\section{Preparation of cis-pinane via $\alpha$-pinene hydrogenation in water by using Ru nanoparticles immobilized in functionalized amphiphilic mesoporous silica $\uparrow$}

\author{
Lihua Xie, ${ }^{a}$ Xiaoyan Wang, ${ }^{a}$ Fengli Yu, ${ }^{a}$ Bing Yuan, (D) a Congxia Xie (D) *a \\ and Shitao Yu iD b
}

Amphiphilic mesoporous silica modified with trimethoxy (3,3,3-trifluoropropyl) silane (TFPS) and (3aminopropyl) trimethoxysilane (APTS) was prepared by one-step synthesis. The structure and morphology of the mesoporous silica were characterized and confirmed by solid-state nuclear magnetic resonance, $\mathrm{X}$-ray diffraction (XRD), $\mathrm{N}_{2}$ adsorption-desorption, Fourier transfer infrared spectroscopy (FTIR), scanning electron microscopy (SEM) and transmission electron microscopy (TEM). Meanwhile, the thermostability of the amphiphilic mesoporous silica was detected by thermogravimetric (TG) technique. Highly dispersed Ru nanoparticles supported on such amphiphilic mesoporous silica (marked as Ru/ MF(MN) were synthesized by wet impregnation method with the assistance of ultrasonic. The catalyst was characterized by X-ray photoelectron spectroscopy (XPS) and inductively coupled plasma-atomic emission spectrometry (ICP-AES). In water medium, Ru/MF $\mathrm{MMN}$ was used in $\alpha$-pinene hydrogenation to prepare cis-pinane. Under the optimum reaction conditions $\left(35^{\circ} \mathrm{C}, 2 \mathrm{MPa} \mathrm{H}_{2}, 1 \mathrm{~h}, \mathrm{~m}\left(\mathrm{H}_{2} \mathrm{O}\right): m(\alpha-\right.$ pinene) $: m(\mathrm{Ru} / \mathrm{MF}(\mathrm{MN})=200: 60: 1), 99.9 \% \alpha$-pinene conversion and $98.9 \%$ cis-pinane selectivity were obtained. When Ru/MF@MN was recycled six times, the conversion slightly decreased and the selectivity was nearly unchanged.

\section{Introduction}

cis-Pinane is a kind of significant industrial intermediate that is usually applied in medicine, materials and perfume owing to the high activity of its $\mathrm{C}_{2}-\mathrm{H}$ bond. ${ }^{\mathbf{1 - 4}}$ cis-Pinane is prepared by selective hydrogenation of $\alpha$-pinene. Traditionally, $\alpha$-pinene hydrogenation is commonly catalyzed by $\mathrm{Pd} / \mathrm{C}^{5}$ or $\mathrm{Pt} / \mathrm{C},{ }^{6}$ but the content of $c i s$-pinane in products is very low. Moreover, carbon deposition or coking tended to occur due to the poor stability of carbon materials when the catalyst was recycled. ${ }^{7}$ Raney-Ni was also commonly used in the hydrogenation of $\alpha$-pinene, but the reaction conditions were too harsh. ${ }^{8}$ When the hydrogenation of $\alpha$-pinene was catalyzed by $\mathrm{RuCl}_{3} \cdot 3 \mathrm{H}_{2} \mathrm{O}$ in water $\left(160{ }^{\circ} \mathrm{C}\right.$, $5 \mathrm{MPa}, 5 \mathrm{~h}$ ), the $\alpha$-pinene conversion could reach $99.7 \%$ and the cis-pinane selectivity was $98.3 \% .^{9}$ However, the stability of the catalyst was very poor.

${ }^{a}$ State Key Laboratory Base of Eco-chemical Engineering, College of Chemistry and Molecular Engineering, Qingdao University of Science and Technology, Qingdao 266042, Shandong, China. E-mail: xiecongxia@126.com; Fax: +86-532-84022719; Tel: +86-532-84022719

${ }^{b}$ College of Chemical Engineering, Qingdao University of Science and Technology, Qingdao 266042, Shandong, China

$\dagger$ Electronic supplementary information (ESI) available. See DOI: 10.1039/c7ra09865k
$\mathrm{Pt},{ }^{10} \mathrm{Pd},{ }^{11} \mathrm{Ni},{ }^{12} \mathrm{Ru}^{13}$ and $\mathrm{Rh}^{14}$ nanocatalysts have been used to catalyze the hydrogenation of $\alpha$-pinene, showing high catalytic activity. However, the agglomeration of metal nanoparticles due to their large surface energy easily results in deactivation of the catalysts. Therefore, stabilizers are commonly needed to prevent the aggregation of metal nanoparticles. $\mathrm{Ru}$ nanoparticles stabilized by methylated cyclodextrin have been applied in the hydrogenation of $\alpha$-pinene at room temperature under 1 bar of $\mathrm{H}_{2}$. The $\alpha$-pinene conversion could reach $100.0 \%$ and $96.0 \%$ of cis-pinane was obtained. ${ }^{15}$ In addition, the selectivity of cis-pinane was improved when $\mathrm{Ru}$ nanoparticles were stabilized with polymeric micelles. ${ }^{16,17}$ However, these nonionic surfactants acted as stabilizers result in the formation of emulsion which consists of the oil-soluble products and the water-soluble catalyst. Therefore, products can not be separated from the emulsion. The above problem could be solved by adding extractant into mixture, but the utilisation of extractant could destroy the structure of micelles and decrease the stability of catalysts. ${ }^{18}$ These results suggest that it is very important to find a suitable catalytic system for $\alpha$-pinene hydrogenation and the catalysts should be easily recycled.

To prevent the formation of aggregates, metallic nanoparticles were commonly loaded on the solid materials such as metallorganics, amorphous silica, activated carbon, magnetic 
materials and mesoporous molecular sieves. ${ }^{\mathbf{1 9 - 2 1}}$ Mesoporous materials are the ideal carrier materials due to the advantages of uniform and adjustable pore structure, large specific surface area and an amount of silicon hydroxyl on the surface of mesoporous materials. Qiu et al. reported that Ni nanoparticles supported on MCM-41 were used to catalyze the hydrogenation of guaiacol at $150{ }^{\circ} \mathrm{C}$ and the conversion was $97.9 \% .{ }^{22}$ Cobalt carbonyl cluster anchored on functionalized SBA-15 showed promising catalytic activity on hydroformylation of 1-octene (97.0\% conversion) with excellent selectivity towards hydroformylated product $(90.0 \%){ }^{23}$ However, these mesoporous materials could not disperse into the organic-aqueous biphase system due to their inherent hydrophilicity in the process of hydrogenation, which made the reaction conditions very harsh.

Micelles are characteristic of the core-shell structure owing to the coexistence of the polar headgroup and the hydrophobic tail. Hydrophilic shell makes micelles evenly dispersed in water while hydrophobic core can solubilize organic substrate. Inspired by the structure of micelles, Yang et al. synthesized amphiphilic mesoporous silica with "micelle-like" structure through the introduction of hydrophilic and hydrophobic functional groups into mesoporous silica. 99.0\% phenol conversion was achieved and the selectivity for cyclohexanone was close to $99.0 \% .^{24-26}$ For our work, Ru nanocatalysts loaded on amphiphilic mesoporous silica were successfully synthesized, and applied in the hydrogenation of $\alpha$-pinene in aqueous medium, exhibiting superior activity and selectivity.

\section{Experimental section}

\subsection{Materials}

Hexadecyltrimethylammonium chloride (CTAC), TMOS, TFPS, APTS, methanol and sodium hydroxide $(\mathrm{NaOH})$ were used in the synthesis of amphiphilic mesoporous silica, and purchased from the Aladdin Industrial Corporation. The metal precursor $\mathrm{RuCl}_{3}$ $\cdot 3 \mathrm{H}_{2} \mathrm{O}$ were obtained from the Aladdin Industrial Corporation. Sodium borohydride $\left(\mathrm{NaBH}_{4}, 98 \%\right)$, ethanol and ethylacetate were supplied by Sinopharm Chemical Reagent Co., Ltd. Hydrogen (99.99 wt\%) was obtained from Qingdao Airichem Specialty Gases \& Chemicals Co., Ltd. $\alpha$-Pinene (98\%) was purchased from Jiangxi Hessence Chemicals Co., Ltd. All chemicals were not purified and used directly. Water was double deionized for use.

\subsection{Preparation of functionalized core-shell mesoporous silica}

Amphiphilic mesoporous silica was prepared by one-step synthesis. Typically, $0.88 \mathrm{~g}$ CTAC, $125 \mathrm{~mL}$ methanol and $625 \mu \mathrm{L}$ $\mathrm{NaOH}\left(1 \mathrm{~mol} \mathrm{~L}^{-1}\right)$ were mixed with $100 \mathrm{~mL}$ water and vigorously stirred at room temperature for $2 \mathrm{~h} .0 .17 \mathrm{~g}$ TMOS and $0.05 \mathrm{~g}$ TFPS were added into the above mixture drop by drop and stirred for $2 \mathrm{~h}$. Then $0.19 \mathrm{~g}$ TMOS and $0.02 \mathrm{~g}$ APTS were dropwise added into the above solution. After that, the solution was further stirred for $12 \mathrm{~h}$ and crystallized for $12 \mathrm{~h}$. The white solid was obtained by filtration and washed to be neutral with water. Then it was dried under vacuum at $50{ }^{\circ} \mathrm{C}$ for $4 \mathrm{~h}$. The solid powder was washed with ethanol 3 cycles (12 h per cycle) to remove excessive CTAC, and dried under vacuum at $50{ }^{\circ} \mathrm{C}$ for $4 \mathrm{~h}$, giving 7.5\%MF@MN (M stands for tetramethoxy-silane (TMOS), F stands for TFPS and $\mathrm{N}$ stands for APTS). Mesoporous silica with different content of fluorine were synthesized according to the above procedures and denoted as 0\%MF@MN, 2.5\%MF@MN and 12.5\%MF@MN, respectively. Moreover, the mesoporous silica without modification $\left(\mathrm{SiO}_{2}\right)$ and that only modified with hydrophobic TFPS (MF@M) were prepared, respectively.

\subsection{Preparation of nanocatalyst}

Ruthenium nanoparticles were embedded in the amphiphilic mesoporous silica by impregnation method. $0.03 \mathrm{~g} \mathrm{RuCl}{ }_{3} \cdot 3 \mathrm{H}_{2} \mathrm{O}$ as metal precursor and $0.20 \mathrm{~g}$ 7.5\%MF@MN were added into $4 \mathrm{~mL}$ ethylacetate and dispersed for 30 minutes under ultrasonic. The mixture was dipped for $12 \mathrm{~h}$ under magnetic stirring at $40{ }^{\circ} \mathrm{C}$. Ethylacetate was removed by centrifugation and another $4 \mathrm{~mL}$ fresh ethylacetate was added. Then, $0.04 \mathrm{~g} \mathrm{NaBH}$ was slowly added into the above mixture under vigorous stirring. After $2 \mathrm{~h}$, the excessive $\mathrm{NaBH}_{4}$ was removed by ethanol and the black precipitate was collected and dried under vacuum at $50{ }^{\circ} \mathrm{C}$ for $4 \mathrm{~h}$, giving $\mathrm{Ru} / \mathrm{MF} @ \mathrm{MN}$. Catalysts denoted as $\mathrm{Ru} / \mathrm{MN}$, $\mathrm{Ru} / \mathrm{SiO}_{2}, \mathrm{Ru} / \mathrm{MF} @ \mathrm{M}$ were synthesized when the carrier was correspondingly replaced by 0\%MF@MN, $\mathrm{SiO}_{2}$ and $\mathrm{MF} @ \mathrm{M}$ (mentioned in Section 2.2), respectively.

\subsection{Selective hydrogenation of $\alpha$-pinene in water medium}

$0.30 \mathrm{~g} \alpha$-pinene, $5 \mathrm{mg} \mathrm{Ru} / \mathrm{MF} @ \mathrm{MN}$ and $1 \mathrm{~mL} \mathrm{H} \mathrm{H}_{2} \mathrm{O}$ were added into a $75 \mathrm{~mL}$ autoclave equipped with a Teflon liner. The autoclave pressurized with $2 \mathrm{MPa}_{2}$ was placed into a $35{ }^{\circ} \mathrm{C}$ water bath under stirring for $1 \mathrm{~h}$. After centrifugation, the upper product phase was collected with a pipette for gas chromatography (GC) analysis.

\subsection{Characterizations}

The morphology of amphiphilic mesoporous silica was determined with a JEOL JSM-6010LV scanning electron microscope and a JEOL JEM-2100 transmission electron microscope. FT-IR spectra were obtained using a Nicolet 510P FT-IR spectrometer with the $\mathrm{KBr}$ method (frequency range from 4000 to $400 \mathrm{~cm}^{-1}$ ). XRD measurements were performed on a Rigaku D/max-2400 diffractometer when $\mathrm{Cu}-\mathrm{K} \alpha$ anode radiation was used as the X-ray source at $40 \mathrm{kV}$ and $100 \mathrm{~mA}$ in the $2 \theta$ range of $0-80^{\circ}$ with a scan speed of $2^{\circ}$ per minute. The porous structure of the catalysts was measured by a Micromeritics ASAP $2020 \mathrm{~N}_{2}$ adsorption-desorption isotherm at $77 \mathrm{~K}$. The content of Ru was determined by using ICP-AES method which was running at $1200 \mathrm{~W}$. Before the analysis, $\mathrm{Ru}$ / MF@MN catalyst was dissolved in a mixture of hydrofluoric acid and aqua regia. The XPS data were recorded by using mono $\mathrm{Al} \mathrm{K \alpha}$ as X-ray source and the hydrocarbon peak of C $1 \mathrm{~s}$ at $284.60 \mathrm{eV}$ was used to calibrate binding energies.

\section{Results and discussion}

\subsection{Synthesis and characterization of mesoporous silica}

The water contact angles of four kinds of mesoporous silica were shown in Fig. 1. Interestingly, water contact angles 


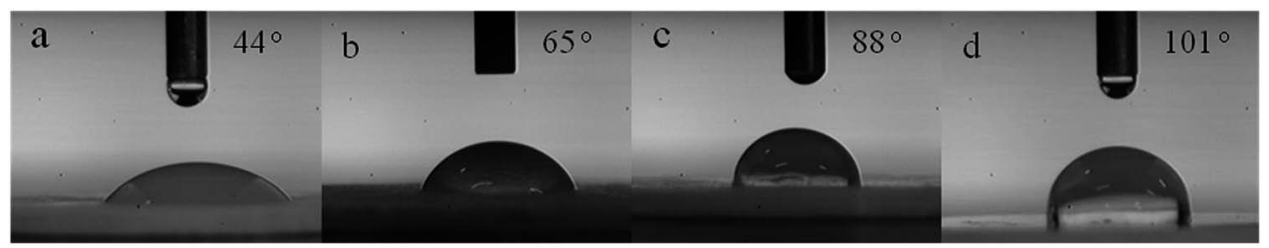

Fig. 1 The images of water contact angles of mesoporous silica: (a) $0 \% \mathrm{MF} @ \mathrm{MN}$, (b) $2.5 \% \mathrm{MF} @ \mathrm{MN}$, (c) $7.5 \% \mathrm{MF}(\mathrm{MN}$ and (d) $12.5 \% \mathrm{MF}(\mathrm{MN}$.
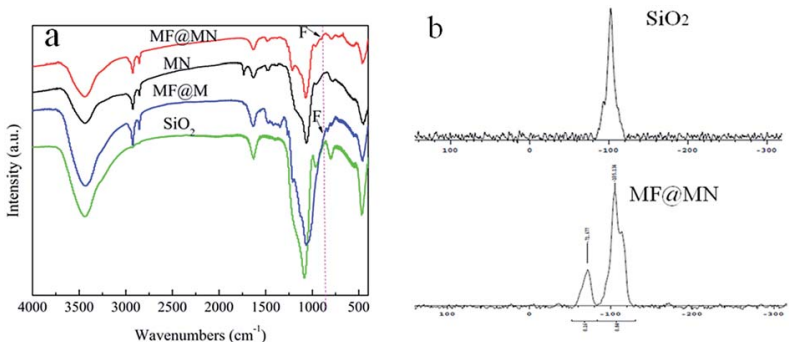

Fig. 2 (a) The FT-IR spectra of MFaMN, MN, MFaM and $\mathrm{SiO}_{2}$, (b) the solid-state ${ }^{29} \mathrm{Si} \mathrm{CP-MAS} \mathrm{NMR} \mathrm{spectra} \mathrm{of} \mathrm{SiO}_{2}$ and MF@MN.

continually increased with an increase in loading of fluorine. The results indicated that the hydrophobicity of mesoporous silica was enhanced. When the content of fluorine increased to $12.5 \%$, the biggest water contact angle was obtained, which meant 12.5\%MF@MN had the strongest hydrophobicity. It resulted in poor dispersity of such mesoporous silica in water. While the content of fluorine was $7.5 \%$, the mesoporous silica with better wettability could disperse well in organic-aqueous biphase system. When Ru nanoparticles were supported on 7.5\%MF@MN, 98.9\% $\alpha$-pinene was converted to cis-pinane and 99.9\% conversion was achieved in water. So 7.5\%MF@MN was selected as the optimum amphiphilic mesoporous silica for the following experiments.

The FT-IR spectra of the as-prepared MF@MN (the same as 7.5\%MF@MN), $\mathrm{MN}$ (the same as 0\%MF@MN), MF@M and $\mathrm{SiO}_{2}$ were shown in Fig. 2a. The peaks appearing at $2960-2855 \mathrm{~cm}^{-1}$ and $2855 \mathrm{~cm}^{-1}$ were attributed to $\mathrm{C}-\mathrm{H}$ stretching vibrations and deformation vibrations. The characteristic peak at $3440 \mathrm{~cm}^{-1}$ was assigned to the contribution of $\mathrm{Si}-\mathrm{OH}$ stretching vibrations. The peak at $1066 \mathrm{~cm}^{-1}$ was attributed to $\mathrm{Si}-\mathrm{O}-\mathrm{Si}$ bending vibrations. The stretching vibrations of $\mathrm{N}-\mathrm{H}\left(3500-3100 \mathrm{~cm}^{-1}\right)$ and $\mathrm{C}-\mathrm{N}$ $\left(1043 \mathrm{~cm}^{-1}\right)$ were not observed which had been overlapped by the stretching vibrations of the $\mathrm{Si}-\mathrm{OH}$ and physically adsorbed water. The peak appearing at $902 \mathrm{~cm}^{-1}$ could be attributed to C-F vibrations. However, for the fluorine-free mesoporous silica MN, this peak was not detected, which indicated that organic silanes had been successfully grafted onto the framework of mesoporous silica.

The solid-state ${ }^{29} \mathrm{Si}$ CP-MAS NMR spectra of $\mathrm{SiO}_{2}$ and MF@MN were shown in Fig. $2 \mathrm{~b}$. The Q-bands assigned to $\mathrm{Si}(-\mathrm{O}-$ $\mathrm{Si}_{x}(\mathrm{OH})_{4-x}$ could be clearly observed. The presence of $\mathrm{Q}^{3}$ and $\mathrm{Q}^{4}$ testified that the framework of mesoporous silica was unchanged..$^{27,28}$ The intense signals for $\mathrm{Q}$ bands confirmed that the mesoporous silica was subjected to a high degree of condensation. Moreover, amphiphilic mesoporous silica with a large number of $\mathrm{Si}-\mathrm{OH}$ was in favor of the successful grafting of organic silane. The presence of T-bands equivalent to $\mathrm{Si}(\mathrm{C})(-\mathrm{O}-$ $\mathrm{Si})_{x}(\mathrm{OH})_{3-x}$ in MF@MN indicated that TFPS and APTS had been incorporated in the mesoporous silica through covalent linkage.

The TG curves of MF@MN and MN were exhibited in Fig. 3. The TG curves revealed that three stages of decomposition occurred for the mesoporous silica. When the temperature was below $160^{\circ} \mathrm{C}$, the weight loss was mainly owing to the physically adsorbed water. When the temperature was between $160^{\circ} \mathrm{C}$ and $300{ }^{\circ} \mathrm{C}$, the weight loss was assigned to the degradation of organic template. When the temperature was higher than $300{ }^{\circ} \mathrm{C}$, the weight loss was mainly due to the decomposition of the grafted TFPS and APTS and the condensation dehydration of silica groups at high temperature. For the mesoporous silica $\mathrm{MN}$, the weight loss was much less than MF@MN, which also confirmed the successful grafting of TFPS.

The pore structure and surface area of mesoporous silica were characterized by $\mathrm{N}_{2}$ adsorption-desorption. The type IV isotherms of both MF@MN and MN could be seen in Fig. 4a, but the hysteresis loop was not obvious when quaternary ammonium salt CTAC was used as the template in the synthesis of mesoporous silica. Compared with $\mathrm{MN}$, the BET specific surface area of MF@MN dropped from 40 to $35 \mathrm{~m}^{2} \mathrm{~g}^{-1}$, the pore volume decreased from 0.111 to $0.103 \mathrm{~cm}^{3} \mathrm{~g}^{-1}$ and the pore size decreased from 2.5 to $2.2 \mathrm{~nm}$, respectively. The above measured values of MF@MN and MN were all smaller than conventional mesoporous sieves without modification. It was mainly because the $\mathrm{N}_{2}$ adsorption-desorption measurements were performed at $-196{ }^{\circ} \mathrm{C}$, so organic silane TFPS and APTS grafted on mesoporous silica became hard and a certain channels were occupied. Perpendicularly oriented channels were observed in Fig. 4c.

Fig. 5 showed the typical XRD patterns of MF@MN, MN, Ru/ MF@MN and Ru/MN. The low-angle XRD patterns of MF@MN and $\mathrm{MN}$ exhibited one diffraction peak in the $2 \theta$ range of $2^{\circ}-3^{\circ}$.

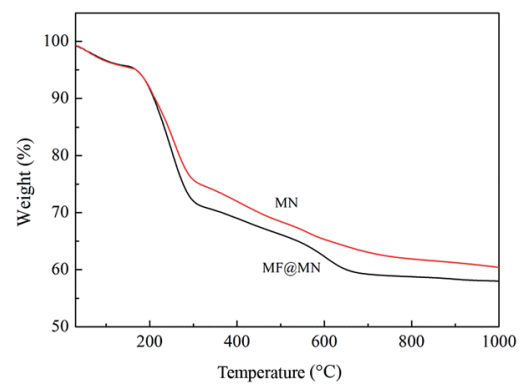

Fig. 3 The TG curves of MF@MN and MN. 

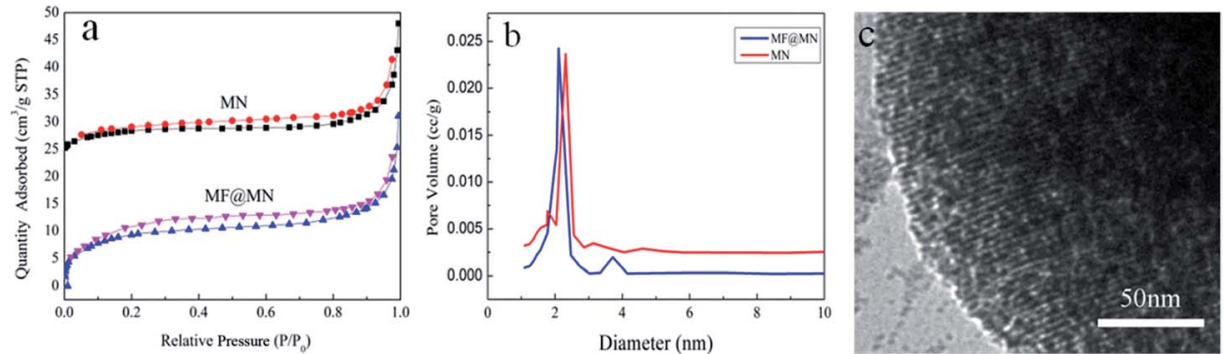

Fig. 4 (a) The $\mathrm{N}_{2}$ adsorption-desorption isotherms, (b) the pore size distribution of MF@MN and MN, (c) the TEM image of MF@MN
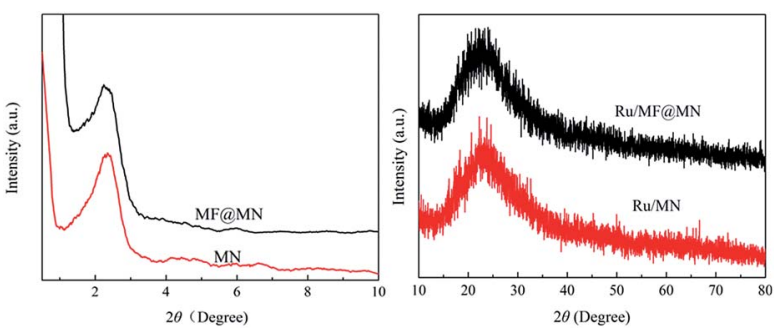

Fig. 5 The XRD patterns of MF@MN, MN, Ru/MF@MN and Ru/MN.

The (100) reflection of amorphous silica confirmed the formation of hexagonal mesoporous structure. The diffraction peak of the amorphous silica was at $22.5^{\circ}$ in high-angle XRD diffraction. However, the diffraction peak at $40^{\circ}$ assigned to Ru nanoparticles was hardly observed. It may be due to the fact that the size of Ru nanoparticles was too small and Ru nanoparticles were dispersed uniformly on surface or in channels of mesoporous silica, ${ }^{29}$ which could be testified by the following TEM images.

Fig. 6 showed the morphology of MF@MN and Ru nanoparticles. The spherical and sheet structure were coexistence when mesoporous silica was synthesized under vigorously stirring as shown in Fig. 6a. In addition, the phenomenon of concatenation among spherical mesoporous silica was observed as well. The mechanical force produced by vigorous stirring was in favor of contact between metastable state methyl

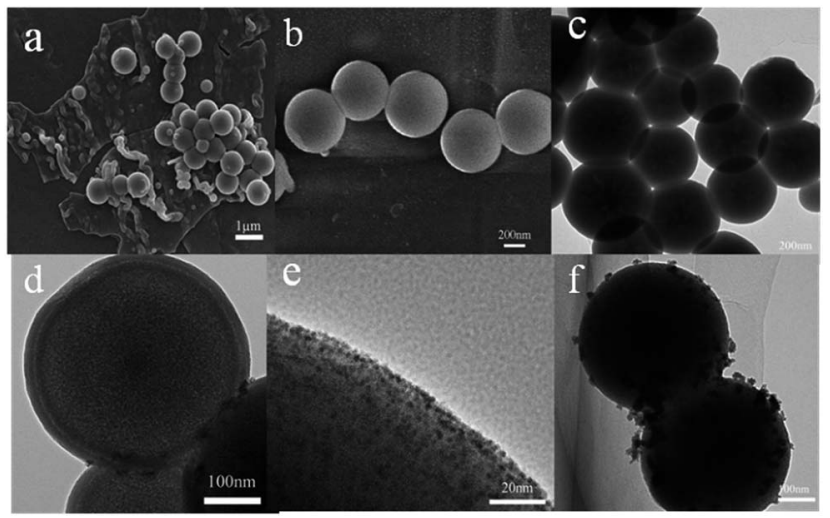

Fig. 6 The morphology of MFaMN and Ru/MF@MN. (a, b) The SEM images of MF@MN, (c) the TEM image of MF@MN, (d, e) the TEM images of Ru/MF@MN, (f) the TEM image of Ru/MF@MN ${ }^{0}$ that was prepared without ultrasonic. orthosilicate TMOS and organic template CTAC, which resulted in the adhesion of nanospheres. The aggregates in emulsion were stretched into flakes or columns owing to the effect of shear stress. ${ }^{30}$ The synthetic conditions of amphiphilic mesoporous silica were optimized such as dispersion time of organic template, content of organic template, $\mathrm{pH}$ of synthetic system, temperature and stirring speed (Fig. S1 $\dagger$ ). Under the optimal conditions, only the uniform spherical mesoporous silica was obtained with an average diameter of $400 \pm 50 \mathrm{~nm}$ as exhibited in Fig. 6b and c. The core-shell structure of spherical mesoporous silica was clearly observed in Fig. $6 \mathrm{~d}$ and the average thickness of the shells was $20 \mathrm{~nm}$ or so.

Scheme 1 depicted the formation of amphiphilic MF@MN and the structure of Ru/MF@MN catalyst. MF@MN contained hydrophobic core (MF) and hydrophilic shell (MN). As can be seen from Fig. 6e, Ru nanoparticles were uniformly immobilized on the surface of MF@MN with an average diameter of $1.8 \mathrm{~nm}$ (Fig. S2†). Fig. 6f was the TEM image of Ru/MF@MN ${ }^{0}$. The catalyst Ru/MF@MN ${ }^{0}$ was prepared by using $\mathrm{MF} @ \mathrm{MN}$ as carrier and Ru nanoparticles were anchored on such amphiphilic mesoporous silica without the assistance of ultrasonic. In Fig. 6f, the aggregates of $\mathrm{Ru}$ nanoparticles were obviously present, which indicated that ultrasonic was in favor of the dispersion of Ru nanoparticles.

In order to deeply analyze the surface elemental composition of the Ru/MF@MN catalyst and valence state of Ru nanoparticles on the MF@MN, the catalyst was detected by XPS technology. These results were shown in Fig. 7. Two peaks centered at $688.5 \mathrm{eV}$ and $402.8 \mathrm{eV}$ in Fig. 7a were assigned to F 1s and N 1s, which indicated that organic silane TFPS and APTS had been grafted to the framework of mesoporous silica successfully. The electron binding energies of $\mathrm{Ru} 3 \mathrm{P}_{1 / 2}, \mathrm{Ru} 3 \mathrm{P}_{3 / 2}$ and $\mathrm{Ru} 3 \mathrm{~d}_{5 / 2}$ were $484.5 \mathrm{eV}, 462.5 \mathrm{eV}$ and $280.4 \mathrm{eV}$, respectively, which were coincident with the standard XPS elemental map of Ru $(484.5 \mathrm{eV}$, $462.0 \mathrm{eV}$ and $280.2 \mathrm{eV}$ ). The enlarged region of $\mathrm{Ru} 3 \mathrm{P}_{3 / 2}$ was shown in Fig. $7 \mathrm{~b}$, the ratio of $\mathrm{Ru}(0)$ was $89.5 \% .10 .5 \%$ ruthenium oxide in Ru/MF@MN was probably due to the oxidation of $\mathrm{Ru}$ nanoparticles during the preparation of samples. ${ }^{31}$

\subsection{The hydrogenation activity of various catalysts and the influence of solvents}

The catalytic hydrogenation of $\alpha$-pinene by various catalysts and the effects of solvents were systematically investigated under the same conditions. The results can be seen from Table 1 . 


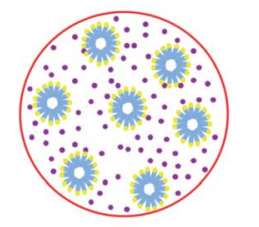

The mixture of $\mathrm{CTAC}, \mathrm{NaOH}$, methanol and water

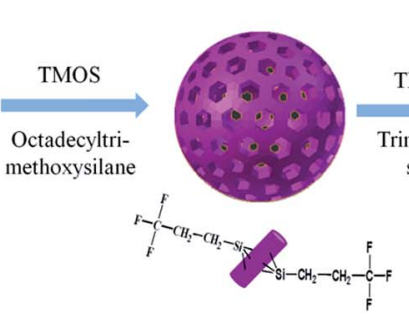

Hydrophobic core (MF)

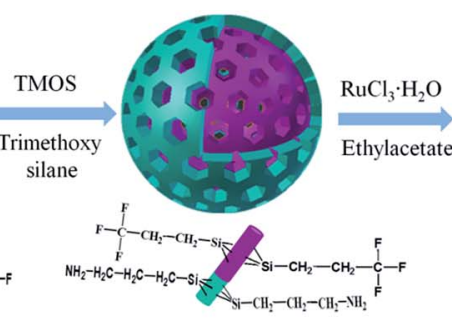

MF@MN

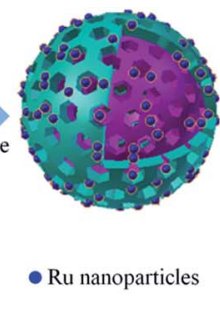

$\mathrm{Ru} / \mathrm{MF} @ \mathrm{MN}$

Scheme 1 Synthesis of amphiphilic mesoporous silica MF@MN and catalyst Ru/MF@MN.
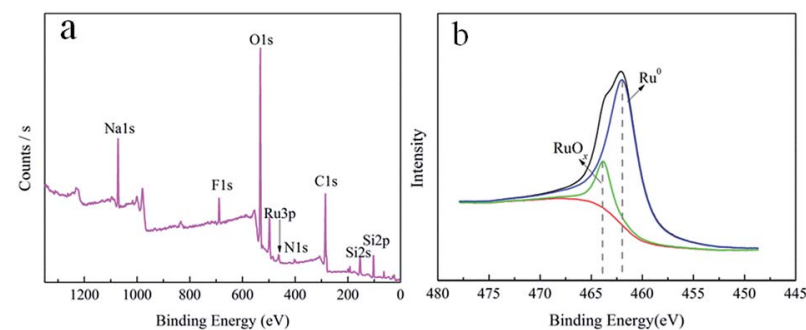

Fig. 7 The XPS spectra of (a) Ru/MF(aMN catalyst and (b) enlarged area of $\mathrm{Ru} 3 \mathrm{P}_{3 / 2}$.

Compared to Raney-Ni and Pd/C, the catalytic activity and cis-pinane selectivity were the highest when $\mathrm{Ru} / \mathrm{C}$ was used in catalytic hydrogenation of $\alpha$-pinene (entries $1-3$ ). So Ru was selected as the catalytic active species in the following experiments. The influence of organic functional groups grafted on the mesoporous silica for hydrogenation of $\alpha$-pinene was studied (entries 4-7). The conversion of $\alpha$-pinene was only $35.7 \%$ when $\mathrm{SiO}_{2}$ was the carrier. The catalyst $\mathrm{Ru} / \mathrm{SiO}_{2}$ (entry 4 ) could not disperse uniformly in organic phase due to its inherent hydrophilicity, which resulted in poor activity of $\mathrm{Ru}$ / $\mathrm{SiO}_{2}$. When the mesoporous silica was only modified with TFPS (marked as MF@M), the catalytic efficiency of catalyst Ru/ MF@M (entry 5) was also poor. It maybe result from the poor

Table 1 The effects of various catalysts and solvents on hydrogenation of $\alpha$-pinene ${ }^{a}$

\begin{tabular}{|c|c|c|c|c|}
\hline Entry & Catalyst & Solvent & Conversion (\%) & Selectivity (\%) \\
\hline 1 & $\mathrm{Ru} / \mathrm{C}$ & $\mathrm{H}_{2} \mathrm{O}$ & $67.9 \pm 0.1$ & $98.7 \pm 0.1$ \\
\hline 2 & $\mathrm{Pd} / \mathrm{C}$ & $\mathrm{H}_{2} \mathrm{O}$ & $68.6 \pm 0.5$ & $87.8 \pm 0.4$ \\
\hline 3 & Raney-Ni & $\mathrm{H}_{2} \mathrm{O}$ & $18.8 \pm 0.4$ & $96.2 \pm 0.3$ \\
\hline 4 & $\mathrm{Ru} / \mathrm{SiO}_{2}$ & $\mathrm{H}_{2} \mathrm{O}$ & $35.7 \pm 0.2$ & $98.5 \pm 0.2$ \\
\hline 5 & Ru/MF@M & $\mathrm{H}_{2} \mathrm{O}$ & $33.1 \pm 0.2$ & $95.8 \pm 0.1$ \\
\hline 6 & $\mathrm{Ru} / \mathrm{MN}$ & $\mathrm{H}_{2} \mathrm{O}$ & $90.2 \pm 0.1$ & $98.5 \pm 0.1$ \\
\hline 7 & Ru/MF@MN & $\mathrm{H}_{2} \mathrm{O}$ & $99.9 \pm 0.1$ & $98.8 \pm 0.1$ \\
\hline 8 & Pd/MF@MN & $\mathrm{H}_{2} \mathrm{O}$ & $98.9 \pm 0.4$ & $80.0 \pm 0.5$ \\
\hline 9 & Ru/MF@MN & $\mathrm{C}_{2} \mathrm{H}_{5} \mathrm{OH}$ & $28.2 \pm 0.2$ & $98.5 \pm 0.1$ \\
\hline 10 & Ru/MF@MN & $\mathrm{C}_{7} \mathrm{H}_{16}$ & $27.0 \pm 0.3$ & $95.8 \pm 0.2$ \\
\hline 11 & Ru/MF@MN & $\mathrm{CH}_{3} \mathrm{COOC}_{2} \mathrm{H}_{5}$ & $30.6 \pm 0.1$ & $99.0 \pm 0.1$ \\
\hline 12 & Ru/MF@MN & - & $3.4 \pm 0.2$ & $98.9 \pm 0.1$ \\
\hline
\end{tabular}

${ }^{a}$ Reaction conditions: $35{ }^{\circ} \mathrm{C}, 2 \mathrm{MPa}_{2}, 500 \mathrm{rpm}, 1 \mathrm{~h}, m\left(\mathrm{H}_{2} \mathrm{O}\right): m(\alpha$ pinene $): m(\mathrm{Ru} / \mathrm{MF} @ \mathrm{MN})=200: 60: 1$. dispersion of Ru/MF@M in water phase because Ru/MF@M possesses strong hydrophobicity. Moreover, the brown solution was observed (Fig. 8a) after hydrogenation of $\alpha$-pinene by $\mathrm{Ru} / \mathrm{MF} @ \mathrm{M}$. It indicated that a part of Ru nanoparticles leached into the product phase. When mesoporous silica was functionalized with hydrophilic APTS (marked as MN), the activity of hydrophilic catalyst Ru/MN (entry 6) was significantly improved and $90.2 \% \alpha$-pinene conversion was obtained. However, the catalytic activity of $\mathrm{Ru} / \mathrm{MN}$ was still lower than that of $\mathrm{Ru}$ / MF@MN (entry 7). When mesoporous silica was modified with both hydrophobic TFPS and hydrophilic APTS (marked as MF@MN), the dispersibility of catalyst Ru/MF@MN in organicaqueous biphasic system was markedly enhanced. Then the amphiphilic catalyst was applied in selective hydrogenation of $\alpha$-pinene. The $\alpha$-pinene conversion could reach as high as 99.9\% and the selectivity for cis-pinane was $98.8 \%$. In addition, the product phase was transparent and colorless after hydrogenation of $\alpha$-pinene (Fig. 8a). It indicated that $\mathrm{Ru}$ nanoparticles existed steadily on the surface of amphiphilic MF@MN. For comparison purpose, Pd nanoparticles loaded on amphiphilic mesoporous silica were prepared by using $\mathrm{Pd}\left(\mathrm{C}_{2} \mathrm{H}_{3} \mathrm{O}_{2}\right)_{2}$ as metal precursor and denoted as Pd/MF@MN. The catalyst was also used in hydrogenation of $\alpha$-pinene. 98.9\% conversion was obtained, but the selectivity for cispinane was only $80.0 \%$ (entry 8 ). It testified the property of ruthenium was in favor of the selective hydrogenation of $\alpha$ pinene compared to Pd species.

The effect of different solvents on hydrogenation of $\alpha$-pinene was also studied (entries 7, 9-12) when Ru/MF@MN was used as catalyst. The difference in catalytic performance was not obvious for organic medium. The conversion was only $30.0 \%$ or so when organic solvents were used. However, in water medium (entry 7), Ru/MF@MN exhibited the best activity and selectivity.

During hydrogenation of $\alpha$-pinene, the uniform $\mathrm{W} / \mathrm{O}$ (denoted as oil-in-water) type bubbles were observed (Fig. 8b). The catalysts acting as solid foaming agent could disperse uniformly in organic-aqueous biphase due to the amphipathy of mesoporous silica (Fig. 8c). The bubbles enlarged the contact area of the solid-liquid-gas triphase and the efficiency of hydrogenation was improved. ${ }^{32}$

Moreover, the "micelle-like" amphiphilic mesoporous silica might be regarded as the microreactors. The hydrophobic core increased the concentration of $\alpha$-pinene and revealed high catalytic activity. The selectivity of $c i s$-pinane could be improved 

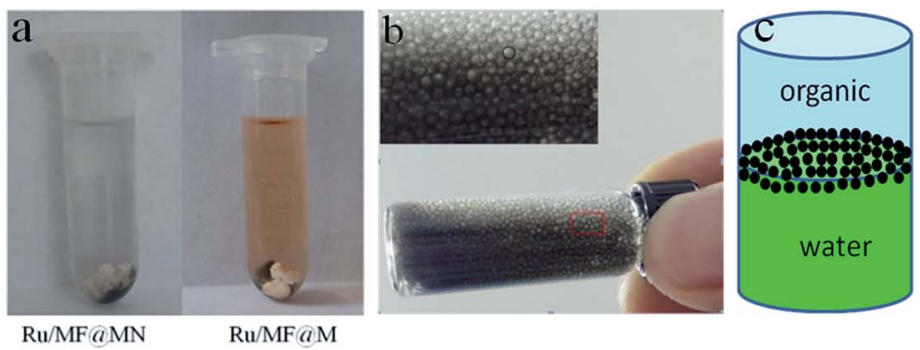

\section{stirring}

Fig. 8 (a) The product phase after hydrogenation of $\alpha$-pinene, (b) the bubbles generated during the hydrogenation of $\alpha$-pinene, (c) the formation mechanism of bubbles.

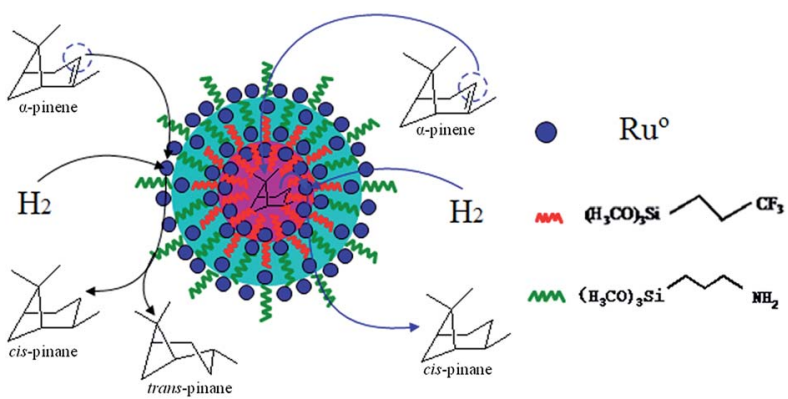

Scheme 2 The hydrogenation mechanism of $\alpha$-pinene.

probably owing to stereoselectivity attack from endo surface of $\alpha$ pinene by catalyst (Scheme 2). ${ }^{33}$ In addition, water was nonpoisonous and harmless for environment and the use of water as solvent was consistent with the environmentally-friendly concept.

When the reaction was performed without $\mathrm{H}_{2} \mathrm{O}$, the catalytic activity of $\mathrm{Ru} / \mathrm{MF} @ \mathrm{MN}$ was very poor, and the $\alpha$-pinene conversion was only $3.4 \%$. It was probably because bubbles and microreactors could not be formed absence of water.

\subsection{Reaction kinetics research}

The kinetics data of hydrogenation of $\alpha$-pinene over $\mathrm{Ru} /$ MF@MN in water were obtained within the temperature range of 298-328 K and under $2 \mathrm{MPa}_{2}$ at $600 \mathrm{rpm}$. When the stirring speed was more than $400 \mathrm{rpm}$, the conversion of $\alpha$ pinene didn't increase with the increase of stirring speed (Fig. S3†). The effect of external diffusion can be ignored at $600 \mathrm{rpm}$ and the reaction enters into the dynamic control area. ${ }^{34}$ Additionally, $2 \mathrm{MPa} \mathrm{H}_{2}$ is adequate for the hydrogenation of $\alpha$ pinene and the catalytic system can be deemed to conform with pseudo-first-order kinetic model. ${ }^{35-37}$

$$
\frac{\mathrm{d}\left(c_{\alpha}\right)}{\mathrm{d} t}=-k c_{\alpha}
$$

The concentration of $\alpha$-pinene $c_{\alpha}$ in eqn (1) is related with the initial concentration $c_{\alpha 0}$ and the conversion of $\alpha$-pinene $x$, which gave eqn (2).

$$
c_{\alpha}=c_{\alpha 0}(1-x)
$$

Hence eqn (1) could be replaced by the following eqn (3).

$$
\frac{\mathrm{d}(1-x)}{\mathrm{d} t}=-k(1-x)
$$

After eqn (3) was integrated against time, eqn (4) was obtained

$$
-\ln (1-x)=k t
$$

The kinetics curves for hydrogenation of $\alpha$-pinene at different temperatures were exhibited in Fig. 9. The linear relation between $-\ln (1-x)$ and time could be observed, which testified that the reaction kinetics of $\alpha$-pinene hydrogenation over Ru/MF@MN was in accordance with pseudo-first-order kinetic model. The slope values of $1.25 \mathrm{~h}^{-1}, 2.33 \mathrm{~h}^{-1}, 5.82$ $h^{-1}$, and $10.15 \mathrm{~h}^{-1}$ are corresponding to the rate constants of $\alpha$ pinene hydrogenation at $25{ }^{\circ} \mathrm{C}, 35{ }^{\circ} \mathrm{C}, 45{ }^{\circ} \mathrm{C}$ and $55{ }^{\circ} \mathrm{C}$, respectively.

The Arrhenius plot of $\alpha$-pinene hydrogenation at different temperatures over Ru/MF@MN is presented (Fig. S4 $\dagger$ ). The apparent activation energy $\left(E_{\mathrm{a}}\right)$ can be figured out according to eqn (5): ${ }^{38}$

$$
\ln k=\ln A-\frac{E_{\mathrm{a}}}{R T}
$$

As shown in eqn (5), the gas constant $R$ is $8.314 \mathrm{~J} \mathrm{~mol}^{-1} \mathrm{~K}^{-1}$. Ea is calculated to be $57.0 \mathrm{~kJ} \mathrm{~mol}^{-1}$.

\subsection{Catalyst recycling}

The reusability of the $\mathrm{Ru} / \mathrm{MF} @ \mathrm{MN}$ system was also investigated. The optimum reaction conditions for hydrogenation of $\alpha$ -

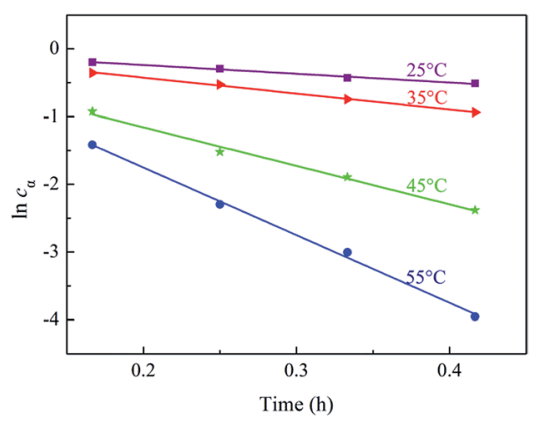

Fig. 9 The plots of $\ln c_{\alpha}$ versus reaction time $t$. 

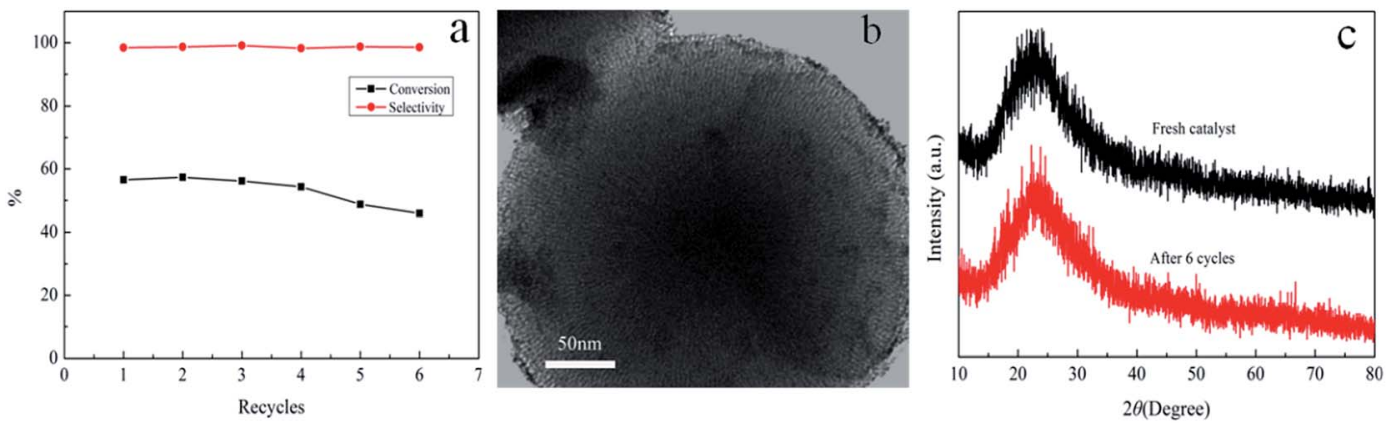

Fig. 10 (a) The reusability of Ru/MFaMN (reaction condition: $35^{\circ} \mathrm{C}, 2 \mathrm{MPa} \mathrm{H}_{2}, 1 \mathrm{~h}, m\left(\mathrm{H}_{2} \mathrm{O}\right): m(\alpha$-pinene) : $m(\mathrm{Ru} / \mathrm{MF}(\mathrm{MN})=330: 100: 1)$, (b) the TEM image of the catalyst after 6 cycles and (c) the XRD patterns of fresh catalyst and catalyst after 6 cycles.

pinene was $35^{\circ} \mathrm{C}, 2 \mathrm{MPa} \mathrm{H}_{2}, 1 \mathrm{~h}, m\left(\mathrm{H}_{2} \mathrm{O}\right): m(\alpha$-pinene $): m(\mathrm{Ru} /$ $\mathrm{MF} @ \mathrm{MN})=200: 60: 1$ (Fig. S5†). After reaction, the catalyst was purged 3 runs by anhydrous ethanol. In addition, it was dried under vacuum at $50{ }^{\circ} \mathrm{C}$ for $4 \mathrm{~h}$. The catalyst could be used directly in the next cycle. When $m\left(\mathrm{H}_{2} \mathrm{O}\right): m$ ( $\alpha$-pinene) $: m(\mathrm{Ru} /$ MF@MN) = 330:100:1, the conversion of $\alpha$-pinene could reach $54.36 \pm 0.1 \%$ and cis-pinane selectivity was $98.71 \pm 0.1 \%$ even after 4 cycles. The invariable conversion was mainly due to the coordination between amino group in amphiphilic shell and Ru nanoparticles. The conversion of $\alpha$-pinene decreased to $45.92 \%$ after six cycles. The TEM and XRD characterization of amphiphilic mesoporous silica after six cycles were shown in Fig. $10 \mathrm{~b}$ and c. It was observed that the morphology and structure of the mesoporous silica were nearly unchanged. The decline of $\alpha$-pinene conversion was probably owing to the leaching of $\mathrm{Ru}$ nanoparticles. Compared to the fresh catalysts, the loss of $\mathrm{Ru}$ nanoparticles was $0.13 \%$ measured by ICP-AES analysis after six cycles. It was mainly because APTS fell off from hydrophilic shell when catalyst was reused. The loss of nitrogen element was $0.35 \%$ according to the results of elemental analysis. The selectivity for cis-pinane was almost unchanged, which perhaps was attributed to the property of $\mathrm{Ru}$ and the shape-selective catalysis of amphiphilic MF@MN.

\section{Conclusions}

The amphiphilic mesoporous silica MF@MN has been prepared by one-step synthesis. A variety of characterizations testify that TFPS and APTS have been successfully grafted on the framework of mesoporous silica. With the assistance of ultrasonic wave, $\mathrm{Ru}$ nanoparticles are uniformly anchored on the surface of amphiphilic mesoporous silica. The Ru/MF@MN catalyst is applied in the selective hydrogenation of $\alpha$-pinene in water medium. Under the optimum reaction conditions ( $35{ }^{\circ} \mathrm{C}, 2 \mathrm{MPa} \mathrm{H}_{2}, 1 \mathrm{~h}, m\left(\mathrm{H}_{2} \mathrm{O}\right): m$ ( $\alpha$-pinene) : $m$ (Ru/MF@MN) $=200: 60: 1$ ), the conversion can reach $99.9 \%$ and $98.9 \%$ of $\alpha$ pinene is converted to cis-pinane. The formation of uniform $\mathrm{W} /$ $\mathrm{O}$ type bubbles in the process of $\alpha$-pinene hydrogenation can enlarge the contact area of the solid-liquid-gas triphase and thus improve the reaction efficiency. The "micelle-like" amphiphilic mesoporous silica can be regarded as the microreactors. Their hydrophobic cores can increase the concentration of $\alpha$-pinene and be beneficial for achieving high catalytic activity. Furthermore, the $\alpha$-pinene conversion and selectivity for cis-pinane are unchanged even after 4 cycles, exhibiting good stability.

\section{Conflicts of interest}

There are no conflicts to declare.

\section{Acknowledgements}

This research was financially supported by the National Natural Science Foundation of China (No. 31270615) and the Taishan Scholars Projects of Shandong Province (ts 201511033).

\section{References}

1 T. Brose, W. Pritzkow and G. Thomas, J. Prakt. Chem., 1992, 334, 403-409.

2 G. Lauterbach and W. Pritzkow, J. Prakt. Chem./Chem.-Ztg., 1995, 337, 416-417.

3 S. Ren, J. Qiu, C. Wang, B. Xu, Y. Fan and Y. Chen, Chin. J. Inorg. Chem., 2007, 23, 1021-1028.

4 V. A. Semikolenov, I. I. Ilyna and I. L. Simakova, Appl. Catal., A, 2001, 211, 91-107.

5 Y. Li, C. G. Pan, W. F. Han, H. F. Chai and H. Z. Liu, Catal. Today, 2011, 174, 97-105.

6 L. Wang, H. Guo, X. Chen, Q. Chen, X. Wei, Y. Ding and B. Zhu, Catal. Sci. Technol., 2015, 5, 3340-3351.

7 M. Opanasenko, P. Stepnicka and J. Cejka, RSC Adv., 2014, 4, 65137-65162.

8 B. I. Mengyu and T. Zeng, J. Nanjing For. Univ., 2003, 511, 115.

9 X. Yang, S. W. Liu, C. X. Xie, S. T. Yu and F. S. Liu, Chin. J. Catal., 2011, 32, 643-646.

10 A. Milewska, A. M. B. Osuna, I. M. Fonseca and M. N. D. Ponte, Green Chem., 2005, 726-732.

11 Y. Yang, X. Liu, D. Yin, Z. Zhang, D. Lei and J. Yang, Russ. J. Appl. Chem., 2015, 26, 333-334.

12 L. Wang, H. Q. Guo, X. P. Chen, Y. Y. Huang and P. P. Zhang, React. Kinet., Mech. Catal., 2015, 114, 639-660. 
13 Y. Yang, X. Liu, D. Yin, Z. Zhang, D. Lei and J. Yang, J. Ind. Eng. Chem., 2015, 26, 333-334.

14 S. Noël, B. Léger, A. Ponchel, K. Philippot, A. DenicourtNowicki, A. Roucoux and E. Monflier, Catal. Today, 2014, 235, 20-32.

15 A. Denicourt-Nowicki, A. Ponchel, E. Monflier and A. Roucoux, Dalton Trans., 2007, 48, 5714-5719.

16 S. L. Hou, X. Y. Wang, C. R. Huang, C. X. Xie and S. T. Yu, Catal. Lett., 2016, 146, 1-7.

17 S. L. Hou, C. X. Xie, F. L. Yu, B. Yuan and S. T. Yu, RSC Adv., 2016, 6, 54806-54811.

18 Y. Liu, L. Li, S. W. Liu, C. X. Xie and S. T. Yu, J. Mol. Catal. A: Chem., 2016, 424, 269-275.

19 Z. Li, R. Yu, J. Huang, Y. Shi, D. Zhang, X. Zhong, D. Wang, Y. Wu and Y. Li, Nat. Commun., 2015, 6, 8248-8255.

20 B. Ghosh, S. Hazra, B. Naik and N. Ghosh, J. Nanosci. Nanotechnol., 2015, 15, 6516-6523.

21 A. Selka, N. Levesque, D. Foucher, O. Clarisse, F. Chemat and M. Touaibia, Org. Process Res. Dev., 2017, 21, 60-64.

22 S. B. Qiu, Y. Xu, Y. J. Weng, L. L. Ma and T. J. Wang, Catalysts, 2016, 6, 134-149.

23 M. Ahmed and A. Sakthivel, J. Mol. Catal. A: Chem., 2016, 424, 85-90.

24 L. Fu, S. Li, Z. Han, H. Liu and H. Yang, Chem. Commun., 2014, 50, 10045-10048.

25 F. W. Zhang and H. Q. Yang, Catal. Sci. Technol., 2014, 5, 572-577.
26 X. F. Li, W. J. Zhang, L. M. Zhang and H. Q. Yang, Chin. J. Catal., 2013, 34, 1192-1200.

27 S. Li, X. Jiao and H. Yang, Langmuir, 2013, 29, 1228-1237.

28 H. X. Wang, H. Q. Yang, H. R. Liu, Y. H. Yu and H. C. Xin, Langmuir, 2013, 29, 6687-6696.

29 F. W. Zhang, S. Chen, H. Li, X. M. Zhang and H. Q. Yang, RSC Adv. , 2015, 5, 102811-102817.

30 D. Liang, Y. Wang, Y. D. Bai and R. F. Li, Chin. J. Inorg. Chem., 2010, 26, 1031-1038.

31 S. L. Hou, C. X. Xie, H. Zhong and S. T. Yu, RSC Adv., 2015, 5, 89552-89558.

32 J. W. Yang, J. M. Duan, F. Daniel and J. Ralston, J. Phys. Chem. B, 2003, 107, 6139-6147.

33 J. M. Brown, A. E. Derome, G. D. Hughes and P. K. Monaghan, Aust. J. Chem., 1992, 45, 143-153.

34 I. L. Simakova, Y. L. Solkina, I. Deliy, A. Wärn, M. Johan and Y. Dmitry, Appl. Catal., A, 2009, 356, 216-224.

35 R. Begum, K. Naseem, E. Ahmed, A. Sharif and Z. H. Farooqi, Colloids Surf., A, 2016, 511, 17-26.

36 F. W. Zhang, S. Chen and H. Li, RSC Adv., 2015, 5, 102811102817.

37 H. Wang, Z. Li and Y. Liu, Green Chem., 2009, 11, 1568-1575. 38 Z. Farooqi, R. Begum, K. Naseem, U. Rubab, M. Usman, A. Khan and A. Ijaz, Russ. J. Phys. Chem. A, 2016, 90, 26002608. 